\title{
Study on Gastrointestinal Evaluation of Iron Deficiency Anaemia Patients Attending at BIRDEM Hospital
}

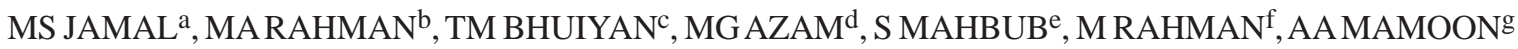

Summery:

Background: Anaemia is common among general population in developing Asian countries. Iron deficiency anaemia (IDA) is the commonest type of anaemia. It is usually due to chronic gastrointestinal blood loss. The standard of care for these patients with IDA includes evaluation of the Gastrointestinal (GI) tract for bleeding lesions. Iron deficiency anemia is considered as an alarm sign for the presence of possible GI malignancies, and inadequate evaluation of patients with IDA may delay the diagnosis of GI tumors especially colorectal cancer.

Objective: To identify the gastrointestinal lesions endoscopically in patients with iron deficiency anaemia. To determine the usefulness of endoscopic procedures (both upper and lower GI) in diagnosis of underlying cause of iron deficiency.

Method: This cross-sectional study was conducted to evaluate Iron deficiency anaemia in patients with or without GI symptoms during the period of July 2010 to December 2010 in the department of Gastroenterology, BIRDEM General Hospital. Sixty eight adult eligible patients with iron deficiency anaemia were taken as per inclusion criteria. All study subjects were underwent endoscopy and colonoscopic procedure after adequate preparation along with examination of their stool. Data were collected through faceto-face interview, observation and document review. Data were recorded and analyzed.

a. Dr. Mohammad Shah Jamal, Assistant Professor, Dept. of Gastroenterology, Comilla Medical College.

b. Prof. Md. Anisur Rahman, Honorary Professor, Dept. of Gastroenterology, BIRDEM General Hospital

c. Dr. Tareq M. Bhuiyan, Associate Professor and Head, Dept. of Gastroenterology, BIRDEM general Hospital

d. Dr. M G Azam, Assistant Professor, Dept. of Gastroenterology, BIRDEM general Hospital

e. Dr. Shawhely Mahbub, Senior Medical Officer, Dept. of ENT and Head \& Neck Surgery, BIRDEM General Hospital

f. Dr. Mahbubur Rahman, Senior Medical Officer, Dept. of Gastroenterology, BIRDEM General Hospital

g. Dr. Abdullah Al Mamoon, Senior Medical Officer, Dept. of Gastroenterology, BIRDEM General Hospital

Address of Correspondence: Dr. Mohammad Shah Jamal, 1/ C, North Kamolapur (1 $1^{\text {st }}$ floor $)$ Dhaka 1217. Phone: 01711133462, E mail: paltu_bd@yahoo.com

Received: 20 January, 2014 Accepted: 12 March, 2015
Results: Majority of patients were 55 to 64 years age group (33.8\%). Mean age \pm SD of this study subject was 54.00 \pm 11.792 with maximum and minimum age 86 and 27 years respectively. More than half of the patients were female (51.5\%) and rests were male 33 (48.5\%). Among the study subjects, $70.58 \%$ patients had GI symptoms, $29.42 \%$ had non-GI symptoms. On stool examination, $17.64 \%$ patients had ova/cyst of helminthes; $82.36 \%$ were normal. Stool OBT revealed $11.8 \%$ positive and $88.2 \%$ negative. On upper GI endoscopy $32.4 \%$ had normal findings, $67.6 \%$ had some lesions. Majority of these lesions were ulcers and erosions (30.9\%), malignancy (ca stomach) was $4.41 \%$; others (which includes congestive gastropathy, reflux oesophagitis, vascular ectasias and helminthiasis) were $32.4 \%$. On colonoscopy, $30.88 \%$ patients had normal colon; $69.12 \%$ had lesions. Among the lesions, most common lesion was hemorrhoids (36.76\%); ca colon was $5.88 \%$ and others (includes ulcers, polyps, vascular ectasias and helminthes) were $26.47 \%$.

Patients with normal upper GI endoscopy- 50\% had GI symptoms and $50 \%$ had non-GI symptoms whereas patients having lesions on upper GI endoscopy $80.4 \%$ had GI symptoms and 19.6\% had non-GI symptoms. This difference was statistically significant $(\boldsymbol{p}<0.05)$.

Patients with normal colonoscopy- $42.9 \%$ had GI symptoms and $57.1 \%$ had non-GI symptoms. On the other hand, patients having lesions on colonoscopy $70.6 \%$ had GI symptoms and $29.4 \%$ had non-GI symptoms. This was also statistically significant.

Conclusion: Majority of the study population had lesions on endoscopy (both upper GI endoscopy and colonoscopy) including malignant lesions. Study showed that lesions are more common in patients with GI symptoms than those without GI symptoms (non-GI symptoms). Therefore, Routine endoscopic (both upper and lower GI) procedures is valuable in evaluating patients with iron deficiency anaemia- for diagnostic as well as therapeutic purposes. Effective treatment of patients with IDA is predicated on the identification of a specific lesion.

(J Banagladesh Coll Phys Surg 2015; 33: 126-132)

Introduction:

Anaemia is common among general population in developing Asian countries. Iron deficiency anaemia (IDA) is the commonest- usually due to chronic gastrointestinal (GI) blood loss when there is no obvious source of bleeding ${ }^{1}$. 
The standard of care for these patients with IDA includes evaluation of the Gastrointestinal (GI) tract for bleeding lesions ${ }^{2}$. Iron deficiency anemia is considered as an alarm sign for the presence of possible GI malignancies, and inadequate evaluation of patients with IDA may delay the diagnosis of GI tumors especially colorectal cancer ${ }^{3}$ In $20 \%$ of patients with IDA a routine upper and lower GI endoscopy may not ascertain GI cause during hospital admission ${ }^{4}$. The available literature, in heterogeneous groups including old age patients and postmenopausal women with IDA, has shown GI lesions in $40-70 \%{ }^{5}$. Studies have shown that increasing age, male gender, ferritin level, prior NSAIDs use, positive fecal occult blood test were factors predictors of endoscopic lesions in patients with IDA with and without GI symptoms ${ }^{6}, 7$. Studies have concluded that prevalence of endoscopic lesions in patients with IDA without GI symptoms is between $48-71 \%^{7}$, however there is a sparse data related to factors predicting GI lesions in this group.

Important implications for recognition of iron deficiency anaemia include diagnosis and treatment of underlying causes, most of which are identifiable by means of conventional upper gastrointestinal endoscopy and colonoscopy $^{8}$. However it remains unresolved which procedure should be done first ${ }^{9}$. Many studies have concluded that on evaluation of Gastrointestinal Tract for IDA; most of the lesions were in lower GI Tract and have recommended that evaluation for IDA should be started with lower GI examination ${ }^{10}$.

There is scanty data to predict the nature and site of GI lesions in IDA patients without gastrointestinal symptoms. Therefore there is a need for studies especially from developing Asian countries, which may establish endoscopic findings and their predictors in this group.

Primary aim of the study was to identify gastrointestinal lesions diagnosed endoscopically in patients with iron deficiency anemia with or without gastrointestinal symptoms.

\section{Method:}

This cross-sectional analytic study was conducted to evaluate Iron deficiency anaemia in patients with or without GI symptoms and their socio-demographic characteristics and attributes associated with GI symptoms. The total period of study was from July 2010 to December 2010. The study was undertaken at the department of Gastrointestinal, Hepatobiliary and Pancreatic disorders (GHPD), BIRDEM. Eligible subjects with Iron deficiency anemia with or without GI symptoms were included: patients admitted in various units of GHPD department, Referred patients from different departments for endoscopy and colonoscopy like Internal medicine, Endocrinology, Nephrology, Surgery and other departments of BIRDEM hospital and Patients seen at gastroenterology outdoor were enrolled after informed consent. All study subjects were underwent endoscopy and colonoscopic procedures after adequate preparation along with examination of their stool. Iron deficiency anemia was defined as hemoglobin concentration $<12.5 \mathrm{~g} / \mathrm{dl}$ for men (normal range, 13.5 to17.5) and <10.6 g/dl for women (normal range, 11.6 to 15.8); With at least one of the following laboratory values consistent with iron deficiency:

- $\operatorname{serum}$ iron $\leq 45 \mu \mathrm{g} / \mathrm{dl}$ (normal range 50 to 150 ) with a transferrin saturation $<10$ percent (normal range 16 to 60 percent),

- $\quad$ serum total iron binding capacity (TIBC) of $\geq 400$ $\mu \mathrm{g} / \mathrm{dl}$ (Normal range 250 - 400)

- Serum ferritin concentration $<20 \mathrm{ng} / \mathrm{ml}$ for men (normal range 20 to 450 ) and $<10 \mathrm{ng} / \mathrm{ml}$ for women (normal range, 10 to 250).

- PBF-microcytic hypochromic anaemia

Estimated sample size was 81. But as in this study purposive sampling technique was used due to the time constraint, 68 samples were taken. After ethical clearance, data were collected by face to face interview using a semi structured questionnaire and from procedures results review. Data were analyzed by SPSS software, cross tabulation, chi square test and independent sample tests.

\section{Result:}

Iron deficiency anaemia is a common and also a serious health problem especially in developing countries. It is often associated with gastrointestinal (GI) lesions. A hospital based cross-sectional study was carried out to 
identify predictors of GI lesions diagnosed endoscopically in patients with iron deficiency anaemia with or without GI symptoms at the department of Gastrointestinal, Hepatobiliary and Pancreatic disorders (GHPD), BIRDEM. Total 68 adult male and female were selected purposively. Data were collected through faceto-face interview, observation and document review. Majority of patients were 55 to 64 years age group (33.8\%). Only 2.9\% patients were in age group 25 to 34 years which got lowest position. Mean age \pm SD of this study subject was $54.00 \pm 11.792$ with maximum and minimum age 86 and 27 years respectively. In this study, more than half of the patients were female (51.5\%) and rests were male 33 (48.5\%). Among the study subjects, $70.58 \%$ patients had GI symptoms, $29.42 \%$ had non-GI symptoms. On stool examination, $17.64 \%$ patients had ova/cyst of helminthes; $82.36 \%$ were normal. Stool OBT revealed $11.8 \%$ positive and $88.2 \%$ negative. On upper GI endoscopy 32.4\% had normal upper GIT, 67.6\% had lesions. Majority of these lesions were ulcers and erosions (30.9\%), malignancy (ca stomach) was 4.41\%; others (which includes congestive gastropathy, reflux oesophagitis, vascular ectasias and helminthiasis) were $32.4 \%$. On colonoscopy, 30.88\% patients were normal colon; $69.12 \%$ had lesions. Among the lesions, most common lesion was hemorrhoids (36.76\%); ca colon was $5.88 \%$ and others (includes ulcers, polyp, vascular ectasias and helminthes) were $26.47 \%$.

Patients with normal upper GI endoscopy- 50\% had GI symptoms and 50\% had non-GI symptoms whereas patients having lesions on upper GI endoscopy 80.4\% had GI symptoms and $19.6 \%$ had non-GI symptoms. This difference was statistically significant $(\mathrm{p}<0.05)$.

Patients with normal colonoscopy- $42.9 \%$ had GI symptoms and $57.1 \%$ had non-GI symptoms. On the other hand, patients having lesions on colonoscopy $70.6 \%$ had GI symptoms and $29.4 \%$ had non-GI symptoms. This was also statistically significant.

Significant (malignant) lesions on upper GI endoscopy were $4.41 \%$ and on colonoscopy was $5.88 \%$. Cross tabulation was done between anaemia and GI symptoms, it was not significant. T test of biochemical analysis and GI symptoms was done, it was also not significant.

Table-I

\section{Age group of the respondents $(N=68)$}

\begin{tabular}{lcc} 
Age group (in Years) & Frequency (n) & Percent (\%) \\
\hline $25-34$ & 02 & 2.9 \\
$35-44$ & 11 & 16.2 \\
$45-54$ & 19 & 27.9 \\
$55-64$ & 23 & 33.8 \\
$>65$ & 13 & 19.1 \\
\hline Total & 68 & 100 \\
\hline
\end{tabular}

Mean \pm SD $54.00 \pm 11.792$, Range- 59, Max- 86, Min- 27

Table-II

\begin{tabular}{lc}
\multicolumn{2}{c}{$\begin{array}{c}\text { Frequency of GI symptoms among the study } \\
\text { subjects ( } N=68)\end{array}$} \\
GI symptoms & Percentage (\%) \\
\hline Abdominal pain & $31.25 \%$ \\
Anorexia & $10.41 \%$ \\
Vomiting & $8.33 \%$ \\
Heart Burn & $16.33 \%$ \\
Loose motion & $12.5 \%$ \\
Dyspepsia & $8.33 \%$ \\
Mucus per rectum & $12.5 \%$ \\
\hline Total & $100.0 \%$ \\
\hline
\end{tabular}

Table-III

Non GI Symptoms among the study subjects $(N=68)$

\begin{tabular}{lc} 
Non GI symptoms & Percentage (\%) \\
\hline Light Headedness & $10.0 \%$ \\
Leg swelling & $15.0 \%$ \\
Breathlessness & $20.0 \%$ \\
G. weakness & $40.0 \%$ \\
Chest pain & $6.0 \%$ \\
Weight loss & $4.0 \%$ \\
Palpitation & $5.0 \%$ \\
\hline Total & $100.0 \%$ \\
\hline
\end{tabular}

Table-IV

GIT status of the patients $(N=68)$

\begin{tabular}{lcc} 
Symptoms & Frequency (n) & Percent (\%) \\
\hline GI Symptom & 48 & 70.58 \\
Non-GI Symptoms & 20 & 29.42 \\
\hline Total & 68 & 100.0 \\
\hline
\end{tabular}




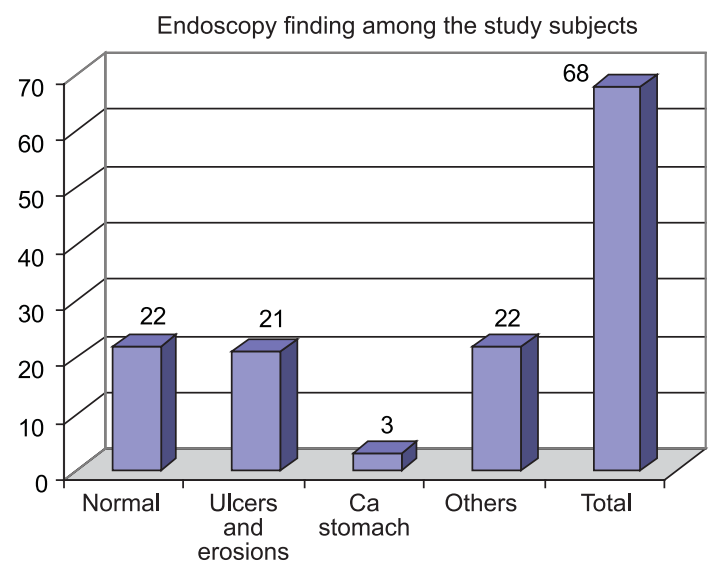

Fig.-1: Endoscopy findings among the study subjects $(N=68)$.

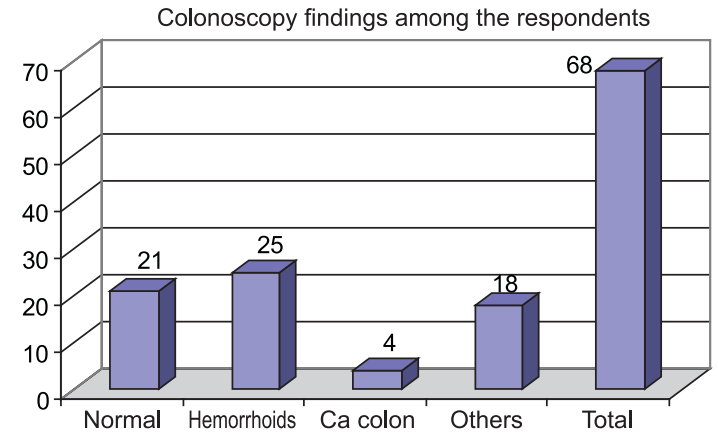

Fig.-2: Colonoscopy findings among the respondents $(N=68)$

Table-V

Findings of Endoscopy and Colonoscopy procedures $(N=68)$

\begin{tabular}{lcc} 
Findings & Endoscopy & Colonoscopy \\
\hline Normal & $22(32.4 \%)$ & $21(30.88 \%)$ \\
Lesions & $6(67.60 \%)$ & $47(69.12 \%)$ \\
\hline
\end{tabular}

Table-VI

\begin{tabular}{lccccc}
\multicolumn{5}{c}{ Chi-square test of endoscopic findings with symptoms (N=68) } \\
Endoscopic findings & GI symptoms & Non-GI symptoms & $\mathrm{x}^{2}$ & $\mathrm{df}$ & $\mathrm{p}$ \\
\hline Normal & $11(50 \%)$ & $11(50 \%)$ & & & 0.01 \\
& & & 6.640 & 1 & \\
\hline Positive findings & $37(80.4)$ & $9(19.6)$ & & & \\
\hline
\end{tabular}

P value $<0.05$, $\mathrm{df}=1, \div 2=6.64$.

Table-VII

Chi-square test of colonoscopy findings and symptoms $(N=68)$

\begin{tabular}{|c|c|c|c|c|c|}
\hline Colonoscopy findings & GI symptoms & Non-GI symptoms & $x^{2}$ & $\mathrm{df}$ & $\mathrm{p}$ \\
\hline Normal & $09(42.9 \%)$ & $12(57.1 \%)$ & \multirow{3}{*}{11.25} & \multirow{3}{*}{1} & \multirow{3}{*}{0.001} \\
\hline & & & & & \\
\hline Positive findings & $39(70.6 \%)$ & 08(29.4\%) & & & \\
\hline
\end{tabular}

$\mathrm{P}$ value $<0.05, \mathrm{df}=1, \div^{2}=11.25$

\section{Discussion:}

Iron deficiency anaemia due to chronic blood loss is usually silent and becomes evident when patients become symptomatic. Studies in patients with IDA without gastrointestinal symptoms are few. It is difficult to conclude what is usual pattern of diseases and factors which can predict the endoscopic out come in IDA patients without gastrointestinal symptoms ${ }^{11}$. 
In this study the respondents aged 25-80 years were selected. The mean \pm SD of age of the respondents was $54 \pm 11.79$ years and $33.8 \%$ respondents were of 55 to 64 age group. Maximum and minimum age were 86 and 27 years where range was 59 years.

More than half of the patients were female 35(51.5\%) and rests were male 33 (48.5\%). Data was mostly collected at working hours which might be the reason for presence of high proportion of female in the sample unit.

Less than one third of the subjects had severe anemia (23.53\%). Most of the subjects had moderate anemia (41.18\%) followed by mild anemia (35.29\%). In a normal population, $2.5 \%$ of the population would be expected to be below this threshold ${ }^{12}$. Hence, iron deficiency anaemia would be considered a public health problem only when the prevalence of haemoglobin concentration exceeds $5.0 \%$ of the population ${ }^{14}$.

Among the study subjects 32.4\% had normal upper GIT at endoscopy, 30.9\% had ulcers and erosions, 36.8\% had other findings such as congestive gastropathy, $\mathrm{Ca}$ stomach, reflux oesophagitis and vascular ectasia.

A study done by Majid et al. showed that $10 \%$ of patients had colonic involvement while $61 \%$ had bleeding and non bleeding causes in upper gastrointestinal tract. Lesions involving both the tracts were present in one patient. Result of this cohort favors that endoscopic evaluation of the upper gastrointestinal tract first ${ }^{15}$.

Normal colonoscopy finding were observed in 30.88\% study subjects, $36.76 \%$ respondents were suffering from hemorrhoids and $32.35 \%$ patients were presented with other GI findings such as Ulcer, Ca colon, Polyp and Vascular ectasia.

Among the study subjects, most of the patients found negative OBT finding (88.2\%). Rest had positive (11.8\%).

In this study, among the patients having normal endoscopic findings- $50.0 \%$ had GI symptoms and rest $50.0 \%$ had non-GI symptoms. Respondents with positive findings at endoscopy $-80.4 \%$ had GI symptom and $19.6 \%$ had non-GI symptoms. These differences were statistically significant $(\mathrm{p}<0.05)$.

Among the patients with normal colonoscopy finding, 42.9\% had GI symptoms and $57.1 \%$ had non-GI symptoms. Among the patients with Positive findings
70.6\% had GI symptoms and 29.4\% had non GI symptoms. This difference was statistically significant $(\mathrm{p}<0.05)$.

Study by Jame et al.,Capurso et al. showed that more than half (70.58\%) of the respondents having GI lesions on endoscopy had gastrointestinal symptom and rests had no such symptoms ${ }^{16}$. The study also showed Hemoglobin, ferritin, female gender and history of NSAIDs have been associated with endoscopic lesions in patients with IDA having gastrointestinal symptoms. In the current study there was no correlation with the factors described in patients with GI symptoms.

Available data in patients with IDA having gastrointestinal symptoms revealed the prevalence of endoscopic lesions is up to $70 \%$ while in asymptomatic IDA patients the cause (bleeding and non-bleeding) was found in $85 \%$ and bleeding related lesions were found in $37-44 \%{ }^{16}$.In another study it was found that $53 \%$ of patients with bleeding related cause for IDA and overall cause of IDA (bleeding and non bleeding lesions) was $71 \%$ on upper and lower GI endoscopy ${ }^{17}$.

A prospective study on patients with iron deficiency aneamia found an upper GIT lesion in $49 \%$ of cases and a lower GIT lesion in $32 \%$ of cases ${ }^{18}$.

Finally, our study demonstrated that endoscopic procedures (both Upper GI endoscopy and Colonoscopy) is valuable in identifying the cause of iron deficiency anaemia in patients with IDA whether they have GI symptom or not. So GIT evaluation is important for every patient with iron deficiency anaemia. Our study also demonstrated those endoscopic lesions (both upper and lower GI) are more common in patients having GI symptom than those without. But, the sample size of this study was small to draw any reasonable conclusions.

\section{Conclusion:}

Essentially any lesion, in any site of the gastrointestinal tract, can bleed in an occult or obscure fashion. The most common manifestation of occult bleeding is iron deficiency anemia. While gastrointestinal tract malignancy is a crucial consideration in this group of patients. In our study, majority of the study population had lesions on endoscopy (both upper GI endoscopy and colonoscopy) including malignant lesions. Study showed that lesions are more common in patients with 
GI symptoms than those without GI symptoms (non-GI symptoms) In this study the most common upper GIT lesion on endoscopy was ulcers and erosions followed by congestive gastropathy, Ca stomach, reflux oesophagitis and vascular ectasia. The most common colonoscopic finding was hemorrhoids followed by other lesions including ulcers, ca colon, polyp and vascular ectasia. Therefore, Routine endoscopic (both upper and lower GI) procedures is valuable in evaluating patients with iron deficiency anaemia- for diagnostic as well as therapeutic purposes. Effective treatment of patients with IDA is predicated on the identification of a specific lesion. Further research is expected to shed light on the role of endoscopy or colonoscopy, in particular to investigate whether these modalities improve outcomes.

\section{Limitations of the study}

Although optimum care had been tried by the researcher in every steps of this study, still some limitations existed:

- The study was conducted in a selected institution. So the study population might not represent the whole community.

- Probability sampling technique could not be employed to recruit the study unit; they were selected purposively due to time constraints. As a result, there might be some selection bias.

- In spite of maximum effort by the researcher due to time and resource limitation sample size was small; a larger sample size would have given a better result.

\section{Recommendations:}

This hospital-based cross sectional study provides information about iron deficiency anaemia and GI symptoms and factors associated with it among adults.

Following recommendations are made based on the study findings:

- When planning therapeutic approaches for IDA patients, GI lesions always should be taken into consideration and identification and treatment of it should be an integral part of IDA management protocol.

- IDA patients, particularly those who are without GI symptom, should be routinely screened for GI lesion

- In this study, small intestine distal to $2^{\text {nd }}$ part of duodenum was not visualized. Lesion in that part causing IDA could not be detected. So, those study subjects who have normal GIT on endoscopy may have lesions in the small intestine. Therefore, evaluation of small intestine by enteroscopy or capsule endoscopy may indentify lesions in those with appearently normal GIT.

- Primary care physicians should be informed that only blood transfusion or iron supplement is not the treatment of iron deficiency anaemaia, rather underlying cause should be found out and treat accordingly.

- Further in depth research should be conducted to clarify the association between GI symptoms and IDA.

\section{References:}

1. Annibale B, Capurso G, Chistolini A, D’Ambra G, DiGiulio E, Monarca B, et al. Gastrointestinal causes of refractory iron deficiency anemia in patients without gastrointestinal symptoms. Am J Med. 2001; 111: 439-45.

2. Ahlquist D, James MW, Chen CM, Goddard WP. Patterns of occult bleeding in asymptomatic colorectal cancer. Cancer. . 1989; 63: 1826-1830.

3. Annibale B. Reversal of iron deficiency anemia after Helicobacter pylori eradication in patients with asymptomatic gastritis. Ann. Intern. Med. 1999; 131: 668-672.

4. Bini E, Baccini F, Osborn J, Panzuto F, Di Giulio E, Delle F, et al. Prospective evaluation of the use and outcome of admission stool guaiac testing: the digital rectal examination on admission to the Medical Service (DREAMS) Study. J. Clin. Gastroenterol. 2006; 40: 821-827.

5. Carey EJ. A single-center experience of 260 consecutive patients undergoing capsule endoscopy for obscure gastrointestinal bleeding. Am. J. Gastroenterol. 2007; 102: 89-95.

6. Ciacci C, Sung IK, Sohn CI, Jeon WK, Kim BI. Helicobacter pylori impair iron absorption in infected individuals. Dig. Liver Dis. . 2004; 36: 455-460.

7. De Leusse. Capsule endoscopy or push enteroscopy for first-line exploration of obscure gastrointestinal bleeding. Gastroenterology. 2007; 132: 855-862.

8. Estevez E, Hopper AN.. Diagnostic yield and clinical outcomes after capsule endoscopy in 100 consecutive patients with obscure gastrointestinal bleeding. Eur. J. Gastroenterol. Hepatol. 2006; 18: 881-888.

9. Gessner B. A controlled, household-randomized, openlabel trial of the effect that treatment of Helicobacter pylori infection has on iron deficiency in children in rural Alaska. J. Infect. Dis. 2006; 193: 537-546. 
10. Guyatt G, Cello JP. Laboratory diagnosis of iron-deficiency anemia: an overview. J. Gen. Intern. Med. 1992; 7: 145-153.

11. Hayashi Y, Cardenas VM, Mulla ZD, Ortiz M, Graham DY. Non-steroidal anti-inflammatory drug-induced small bowel injuries identified by double-balloon endoscopy. World J. Gastroenterol. 2005; 11: 4861-4864.

12. Huprich J, Mandal AK, Mehdi I, Munshi SK, Lo TC E, Mulla ZD, et al. Obscure gastrointestinal bleeding: evaluation with 64-section multiphase CT enterographyinitial experience. Radiology. 2008; 246: 562-571.

13. James MW, Binni F. Risk factors for gastrointestinal malignancy in patients with iron-deficiency anaemia. Eur J Gastroenterol Hepatol. 2005; 17(11):1197-203.
14. Jolobe O. Guidelines for the management of iron deficiency anaemia Gut. 2001; 49:158-164.

15. Kaye P. The clinical utility and diagnostic yield of routine gastric biopsies in the investigation of iron deficiency anemia: a case-control study. Am. J. Gastroenterol. 2008; 103: 2883-2889.

16. Leighton JA, Kaye P. Obscure gastrointestinal bleeding. Gastrointest. Endosc. 2003; 58: 650-655.

17. Lai LH Goh KL, Quek KF, Yeo GT, Hilmi IN. Long-term follow-up of patients with obscure gastrointestinal bleeding after negative capsule endoscopy. Am. J. Gastroenterol. 2006; 101: 1224-1228.

18. Looker AC, Dallman PR, Carroll MD, Gunter EW, Johnson CL. Prevalence of iron deficiency in the United States. JAMA. 1997; 277: 973-976. 